\title{
Comparison of the Core Flow Solution and the Full Solution for MHD Flow
}

\author{
L. Lenhart, K. McCarthy \\ Institut für Reaktorbauelemente \\ Projekt Kernfusion
}

\section{Kernforschungszentrum Karlsruhe}




\section{KERNFORSCHUNGSZENTRUM KARLSRUHE \\ Institut für Reaktorbauelemente \\ Projekt Kernfusion}

KfK 4681

Comparison of the Core Flow Solution and the Full Solution for MHD Flow

L. Lenhart, K. McCarthy

Kernforschungszentrum Karlsruhe $\mathrm{GmbH}$, Karlsruhe 
Als Manuskript vervielfältigt

Für diesen Bericht behalten wir uns alle Rechte vor

Kernforschungszentrum Karlsruhe $\mathrm{GmbH}$ Postfach 3640,7500 Karlsruhe 1

ISSN 0303-4003 


\section{Vergleich von Kernströmungs-Lösung und voller Lösung für MHD-Strömung}

Das selbstgekühlte Flüssigmetall-Blanket ist eines der Konzepte für den Einsatz in Fusions-Reaktoren. Die Präsenz starker Magnetfelder beeinflußt die Wärmeabfuhr, den Massenstrom und den Druckabfall. Für den Nachweis der Machbarkeit spezieller Blankets müssen daher diese grundlegenden Komplexe untersucht werden.

Detailierte Informationen liefert die Lösung des vollen Satzes der nichtlinearen dreidimensionalen MHD-Gleichungen. Weil dies bei nicht voll eingelaufener Strömung hohe Rechenzeiten und großen Speicherplatzbedarf impliziert, ist eine alternative Lösungsmethode wünschenswert. Eine dieser Methoden nutzt die Tatsache, daß Reibungs- und Trägheitseinflüsse bei großen Hartmannzahlen $M$ und Interaktionsparametern $N$ vernachlässigbar sind. Besondere Charakteristiken der resultierenden linearen Gleichungen erlauben den Einsatz zweidimensionaler Rechenprogramme, ohne dabei die dreidimensionale Information zu verlieren. Um den Gültigkeitsbereich dieser "core flow solution" (CFS) bezüglich $M$ und $N$ zu bestimmen, werden die Ergebnisse mit der "full solution" (FS) für eine Strömung im Rechteckkanal bei variablem senk- rechten Magnetfeld verglichen. Während beide Verfahren stationäre $\mathrm{Zu}$ stände annehmen und das induzierte Magnetfeld vernachlässigen, berücksichtigt nur die FS Reibungs- und Trägheitsterme.

Vergleiche werden für verschiedene $M$ und $N$ durchgeführt, wobei der Speicherplatz und somit die Maschen-Reynoldszahl deren Kombination für die FS determinieren. Die Ergebnisse beider Verfahren für Geschwindigkeit, Potential und Druck zeigen akzeptable Übereinstimmungen, und die Kerngeschwindigkeiten differieren bei $M \geq$ 200 um weniger als ein Prozent. Unterschiede der Geschwindigkeitsprofile und Potentiale treten in Wandnähe auf, weil die CFS dort keine exakten Ergebnisse liefern kann. Die FS errechnet dagegen das typische M-Profil für voll eingelaufene MHD-Strömung und eine gewöhnliche hydrodynamische Verteilung im Bereich kleiner Magnetfelder. Wegen der genauen Bestimmung des Volumenstromes kann die CFS zur Vorhersage des Wärmetransportes herangezogen werden, wohingegen die FS auch bei Überlegungen zur Korrosion eingesetzt werden kann. Die Variation der hier realisierbaren Interaktionsparameter für FS, $N \geq 100$ 
bei $M=50$ und $N \geq 2000$ bei $M=300$ hat keinen Einfluß auf die Resultate.

Die CFS ist erheblich schneller als die FS und beansprucht weniger Speicherplatz, wenn die Gleichungen auf zwei Dimensionen reduziert werden. Daher sollte dieses Verfahren, dort wo es anwendbar ist, den Vorzug erhalten. Weitere Vergleiche bei niedrigerem $\mathrm{N}$ und komplexeren Geometrien, wie Umlenkungen und sprunghafte Querschnittsveränderungen, wären aufschlußreich, weil in diesen Fällen Reibung und Trägheit von größerem Einfluß sind. 


\section{Abstract}

The self cooled liquid metal blanket is a prime candidate for use in a fusion reactor. The presence of a magnetic field affects heat transfer, mass transfer and pressure drop. Therefore it is important to analyse general features of a blanket in order to assess the viability of a particular blanket.

The most detailed informations can be obtained by solving the full set of three-dimensional nonlinear MHD equations, but for other than fully developed flow this can take extensive computer time and storage. It is therefore desirable to use an alternative method to predict the flow variables. One such method is based on the fact that viscous and inertial terms are negligible if the Hartmann number $M$ and the interaction parameter $\mathrm{N}$ are high enough. Then certain characteristics of the resulting linear equations allow the use of a two-dimensional code without losing the three-dimensional information. In order establish the range of $M$ and $N$ that the core flow solution (CFS) is valid, the results are compared to the full solution (FS) of a flow in a rectangular duct with a varying perpendicular magnetic field. Both codes assume steady state conditions, and neglect the induced magnetic field. Only the FS includes inertial and viscous terms.
Comparisons are made at a few different Hartmann numbers and interaction parameters. Computer storage and thereby the mesh Reynolds number determine the combination of $M$ and $N$ used in the FS code. The results show similar trends in velocities, potentials and pressures. The quantitative agreement is within acceptable error limits, and the calculated core flow velocities differ less than one percent for $M \geq$ 200. Differences in the velocity profile near the walls and in the potentials near the side walls are evident, because the CFS does not yield exact velocities in the boundary layers and neglects the jump of the potentials across the side layers. The FS calculates the typical M-shaped profile for fully developed MHD flow and an ordinary hydrodynamic distributions where the magnetic field is low. Since the CFS provides a good estimation of the volume flux it can be used to predict heat transfer, whereas the FS also can be used for corrosion analysis. All interaction parameters available for the FS, $N \geq 100$ for $M=50$ and $N \geq$ 2000 for $M=300$, do not effect the results significantly.

The CFS is much faster than the FS, and, if the equations are reduced to two dimensions, the numerical sol- 
ution takes much less computer storage. Therefore this method should be prefered when applicable. Further comparisons should be done at lower interaction parameters and in more complex geometries such as bends or abrupt expansions, where inertial forces and viscosity may have a greater effect. 


\section{Table of Contents}

1. Introduction

2. The different sets of equations .. 2

2.1 Equations of the full solution (FS) $\ldots \ldots \ldots \ldots \ldots \ldots \ldots$

2.2 Equations of the core flow solution (CFS) ............ 3

3. Algorithm ........... 5

3.1 Full solution (FS) $\ldots \ldots \ldots 6$
3.2 Core flow solution (CFS) . . . 7

4. Numerical results $\ldots \ldots \ldots .9$

5. Conclusions .......... 21

Appendix A. Nomenclature . . . 22

Literature $\ldots \ldots \ldots \ldots . . .23$ 


\section{List of Illustrations}

Figure 1. Geometry of the duct .. 5

Figure 2. Results for $M=50$, $N=2000, c=0.07 \quad \ldots 10$

Figure 3. Results for $M=100$, $N=2000, c=0.07 \ldots 11$

Figure 4. Results for $M=300$, $\mathrm{N}=2000, \mathrm{c}=0.07$ 12

Figure 5. Development of velocity distribution for CFS (3-D plot)

Figure 6. Development of velocity distribution for FS (3-D plot) . . . . . . . 14

Figure 7 . Potential in the $y-z-$ plane at $y=0$ and $x=-7.5$ 15

Figure 8 . Current in the $x$-z-plane at $y=0 \ldots \ldots \ldots 16$

Figure 9. Comparison of the pressure gradient . . . 17

Figure 10. Comparison of the core velocity ........ 18

Figure 11. FS with $M=100$, $c=0.07$, various $N \ldots 20$ 


\section{Introduction}

The examination of the flow of a conducting fluid under the influence of a strong magnetic field is essential for the design of a self cooled liquid metal blanket. In a fusion reactor, the self cooled blanket is responsible for both heat transfer and tritium breeding simplifying the blanket structure, which is one of the reasons that it is a prime candidate for use. In order to determine the feasibility of the liquid metal blankets, the velocity distribution and the pressure losses of the fluid must be predictable. To reach this goal, it is always desirable to have experimental and theoretical studies ( see for example / 2, 3, 4/). Because analytical methods such as asymptotic expansions are not always efficient or possi- ble for all reactor relevant applications, the theoretical analysis is done through development of numerical schemes.

This paper compares two methods used to predict the three-dimensional magnetohydrodynamic (MHD) flow of a conducting fluid in a rectangular duct under the influence of a varying magnetic field. The main difference between them is, in general terms, that one of them neglects viscous and inertial effects to simplify the equations, and thereby obtains solutions much faster. As a consequence of these approximations, the range of application is restricted, and a check of the validity of the results is required. 


\section{The different sets of equations}

The two different models studied here, the full solution (FS) and the core flow solution ( CFS ), refer to different sets of equations. The FS is more general, but as a result much more programming effort, computational time and storage is required. Particularly because the storage of modern vector computers is still limited, it is desirable to use an easier alternative method, such as the CFS, for predicting the flow variables.

\subsection{Equations of the full solution (FS)}

Magnetic fields in fusion reactors are slowly varying, and the magnetic Reynolds number $R e_{m}$, the ratio of induced to applied field, is small. As a result, the induced magnetic field is often negligible. The typical MHD flow in this case can be assumed inductionless, incompressible and isothermal. The isothermal approximation makes the buoyancy forces negligible and decouples the Navier-Stokes equation from the conservation of energy. Thereby the flow variables can be computed without solving the energy equation simultaneously. Considering all these approximations, the full set of dimensionless equations describing nonrelativistic MHD flow consists of the Navier-Stokes equation, Maxwell's equations, conservation of mass, conservation of electric charge and Ohm's law. They can be written in the operational form $/ 1 /$ :

Navier-Stokes equation

$$
\begin{aligned}
& \frac{1}{N}\left\{\partial_{\mathrm{t}} v+(\mathrm{v} \nabla) v\right\} \\
& =-\nabla p+\mathbf{j} \times \mathrm{B}+\frac{1}{\mathrm{M}^{2}} \Delta \mathbf{v}
\end{aligned}
$$

conservation of mass

$$
\nabla \cdot v=0
$$

conservation of electric charge

$$
\Delta \Phi=\nabla \cdot(\mathrm{v} \times \mathrm{B})=\mathrm{B} \nabla \times \mathrm{v}
$$

Ohm's law

$$
\mathbf{j}=-\nabla \Phi+\mathbf{v} \times \mathbf{B}
$$

where $t, v, p, j, B, \Phi$ are the dimensionless time, velocity, pressure, current density, magnetic field and electric potential, nondimensionalized by 
$a / v_{0}, v_{0}, \rho_{0} v_{0}{ }^{2} \mathrm{~N}, \sigma v_{0} \mathrm{~B}_{0}, \mathrm{~B}_{0}, a v_{0} \mathrm{~B}_{0}$, respectively. $\mathrm{B}_{\mathrm{O}}$ is the applied magnetic field, a is a characteristic length (in this case the half width of the rectangular channel), $v_{0}$ the average velocity, $\sigma$ the fluid's conductivity and $\rho_{0}$ the fluid's density. The Hartmann number $\mathrm{M}=\mathrm{aB}_{\mathrm{O}}(\sigma / \eta)^{1 / 2}$ gives the square root of the ratio of electromagnetic forces to viscous forces. Here $\eta$ is the viscosity of the fluid. $\mathrm{N}=\sigma \mathrm{aB}_{\mathrm{o}}{ }^{2} /\left(\rho_{\mathrm{o}} \mathrm{v}_{\mathrm{o}}\right)$ is the interaction parameter, giving the ratio of electromagnetic forces to inertial forces.

The obligatory boundary conditions are the no-slip condition at each wall of the channel

$$
\left.v\right|_{\text {wall }}=0 \text {, }
$$

and the thin wall approximation, as introduced by Shercliff / $7 /$ and later made more general by Walker / 8 /:

$$
\partial_{n} \phi I_{\text {wall }}=c \Delta_{t} \phi I_{\text {wall }}
$$

Here $\mathrm{c}=\sigma_{\mathrm{w}} \mathrm{t}_{\mathrm{w}} / \sigma \mathrm{a}$ is the wall conductance ratio, giving the ratio of wall conductance to fluid conductance, $\sigma_{\mathrm{w}}$ and $t_{w}$ being the conductivity of the wall and its thickness. $\partial_{n}$ is the normal derivative pointing into the wall and $\Delta_{t}$ is the tangential part of the Laplacian operator. Applying the thin wall approximation, the flow through the channel can be simulated without solving Maxwell's equations in the wall. The Hartmann number, interaction parameter and conductance ratio are the dimensionless numbers characteristic of MHD flow. Typical values for fusion blankets are $10^{3} \leq \mathrm{M}$ $\leq 10^{4}, 10^{2} \leq \mathrm{N} \leq 10^{4}$ and $1 / 10^{3} \leq \mathrm{c} \leq$ $1 / 10^{2} / 10,11 /$. Because $M$ and $N$ are so large, inertial effects and friction are generally confined to thin layers with steep velocity gradients. Numerical simulation of these thin shear layers is difficult, because they must be resolved properly. On the other hand, the greater part of the flow, i.e. the whole core, is determined by a dominating Lorentz force $\mathbf{j} \times \mathrm{B}_{0}$. These two aspects lead to the CFS which calculates the variables in the core and provides the non-negligible flow carried by the side layers. The exact description of the flow variables in the thin boundary layers is assumed to be of minor importance then, in order to find out the relevant characteristics and variables of the MHD core flow.

\subsection{Equations of the core flow solution (CFS)}

The idea of the CFS is to neglect the nonlinear inertial terms and the viscous effects which make the numerical scheme of the FS difficult. This is rea- 
sonable if $N$ and $M$ are high enough, as can be seen from equation [2.1]. Then only the Lorentz force balances the pressure gradient, and the governing dimensionless equations, representing the core flow, read / 8 /:

Navier-Stokes equation

$$
\nabla p=\mathbf{j} \times \mathbf{B}
$$

conservation of mass

$$
\nabla \cdot v=0
$$

conservation of electric charge

$$
\nabla \cdot \mathbf{j}=0
$$

Ohm's law

$$
\mathbf{j}=-\nabla \Phi+\mathbf{v} \times \mathbf{B} .
$$

Notice that neither the Hartmann number nor the interaction parameter appear in this set of linear equations, but still the same number of variables is involved. Because the viscosity is assumed to be negligible, the no-slip condition at the duct's wall cannot be satisfied with this set of equations. The new boundary condition then requires the normal component of the velocity to vanish at each wall,

$$
\left.v_{n}\right|_{\text {wall }}=0
$$

The thin wall approximation of $F S$ and CFS are the same:

$$
\left.\partial_{n} \Phi\right|_{\text {wall }}=\left.c \Delta_{t} \Phi\right|_{\text {wall }} .
$$




\section{Algorithm}

A rectangular duct as shown in figure 1 is examined. Although it is possible, the conductance ratio is not considered to be variable in this example for means of simplicity. A constant value typical of a fusion reactor is chosen. Cartesian coordinates are used with $x$ pointing in the main stream direction. The cross section of the square duct extends from $-1 \leq y \leq 1$ and $-1 \leq z \leq$
1 , whereas the axial domain is $-15 \leq x$ $\leq 15$. A variable magnetic field

$$
B_{y}(x)=\frac{0.95}{1+e^{0.75 x}}+0.05
$$

is applied with the subscript indicating its direction.

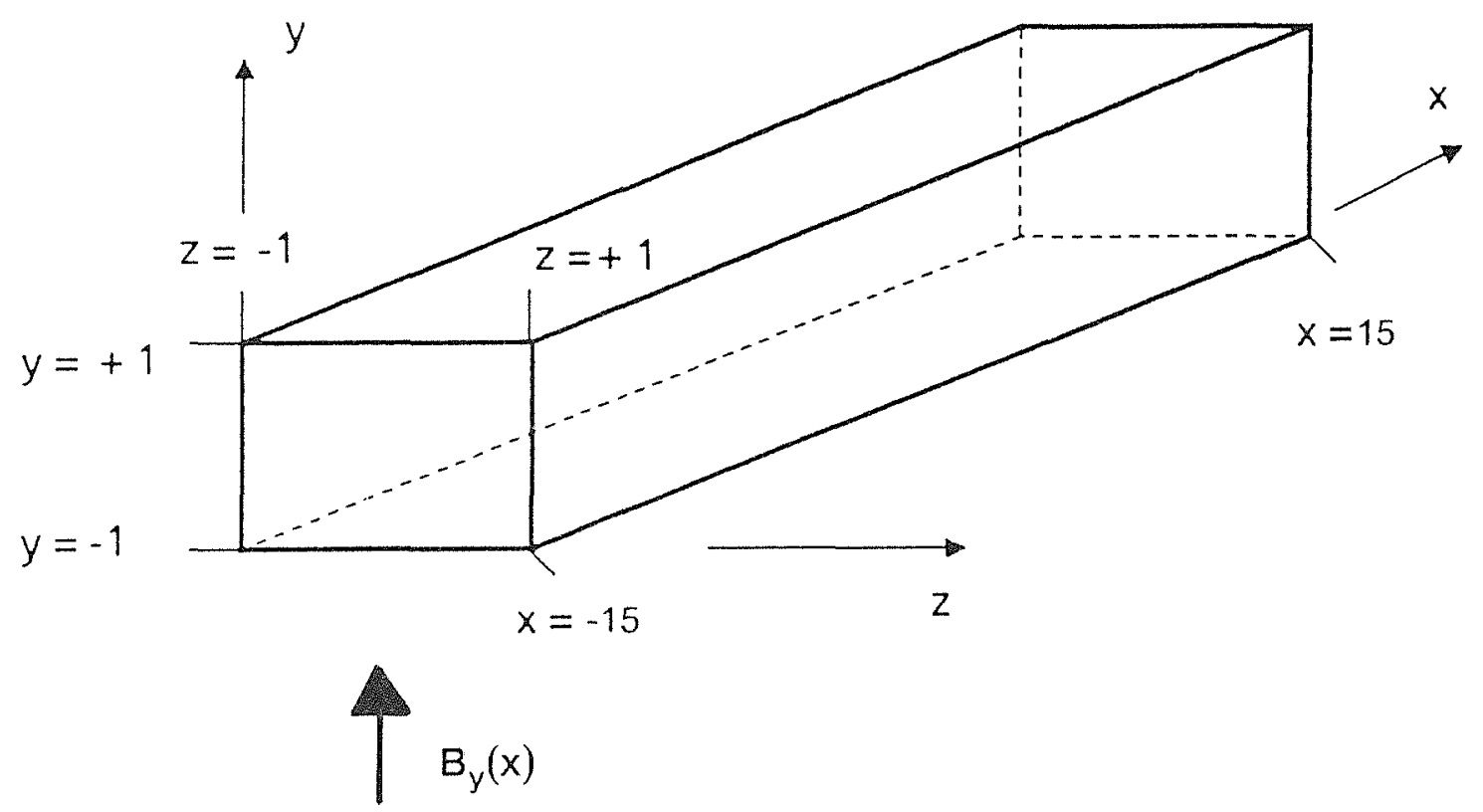

Figure 1. Geometry of the duct 


\subsection{Full solution (FS)}

The set of equations shown in chapter 2.1 are evaluated with a vectorized version of the numerical method described in $/ 1 /$. A staggered grid discretises the computational domain defining the scalars $p$ and $\phi$ in the middle of the cell, and the flux variables $j$ and $v$ in the middle of the sides. This grid generation is chosen to ensure mass and current conservation. A linear interpolation of the variables is employed to figure out values where they are not defined. Equidistant central differences with second order accuracy are applied. After an initial guess for the three velocity components, the governing equations are advanced forward in time until the steady-state solution is reached. A time splitting procedure is used for the time integration. The integration of the Navier-Stokes equation is performed by an ADI method. Two Poisson-type equations for electric potential and pressure, respectively, have to be solved each time step with a fast Poisson solver.

The storage available on the VP50 computer in KfK limits the discretisation to about $70 \times 62 \times 62$ nodes in $\times$-, $y$ - and z-direction, respectively. The gradients of the velocity profile in the layers close to the wall increase at higher Hartmann numbers. In a rec- tangular duct the thickness of the side layers at the walls parallel to the magnetic field lines is proportional $\mathrm{M}^{-1 / 2}$, the one of the Hartmann layers at the walls perpendicular to the magnetic field lines is proportional $M^{-1}$. Because velocity variations have to be resolved properly, there is a upper limit of the Hartmann number depending on the number of grid points and the magnetic field. Steeper gradients of B produce steeper gradients of the velocity. For the variable magnetic field chosen here and the storage available on the VP50 the maximum of $M$ is restricted to about 300 .

As shown for example in / 5/, there is another restriction for numerical calculations with the FS. If the relation for the mesh Reynolds number

$$
\frac{M^{2}}{N} \Delta x U \leq 2
$$

is not satisfied, the validity of the solution is not guaranteed. Here $\Delta x$ is the dimensionless distance between two neighbouring grid points, and $U$ is the local dimensionless velocity. This relation yields a minimum interaction parameter, due to the maximum number of grid points determined by computer storage and the applied magnetic field. A faster variation of $B$ enhances the local velocity $U$ in the side layers. If $\mathrm{N}$ is too low the calcu- 
lation can become inconsistant and yields results alternating at every computational time step. The minimum interaction parameter for a magnetic field defined in equation [3.1] and a Hartmann number of 50 is about 100 . For $M=300$ the minimum $N$ increases to about 2000 .

The typical computational time for the FS depends on the number of nodes and the parameters chosen. With $34 \mathrm{x}$ $34 \times 34$ grid points the cpu-time for a Hartmann number of 50 and an interaction parameter of 2000 is a few minutes, rising to more than 15 hours for $M=300$ with $62 \times 62 \times 62$ grid points. This cpu-time can be reduced by a factor of five to ten if a "good" guess for the initial velocities is done. For example the results at a lower Hartmann number can be taken as initial values for calculations with a higher Hartmann number. If the applied magnetic field and the interaction parameter is not changed, convergence is reached much faster then.

\subsection{Core flow solution (CFS)}

The set of equations shown in chapter 2.2 can be solved in the present form with a three-dimensional computer code. The equations are linear and therefore easier to solve than the set of nonlinear equations of the FS. But
MHD flows at high Hartmann numbers and interaction parameters have special characteristics that can be taken advantage of. The basic equations can be used to show that the pressure and the components of the current density perpendicular to the applied field do not vary along the magnetic field lines. The equations are integrated first along these lines analytically. Once this procedure is done, the equations can be solved on the two-dimensional surface numerically without losing the three-dimensional information of the entire domain / 2 /. After the variables are known on the surface, the additional variables of the three-dimensional duct flow can be derived, because their variation along field lines is known. This reduces the computer time needed, the code complexity and the storage required drastically, whereas the analytical preliminaries imply more effort.

After an initial guess for the core flow velocity, SOR is employed to calculate the potential distribution in the fluid and the wall. As long as the values for the potential do not vary along field lines in a prescribed manner, the velocities are adjusted and normalized, and the procedure is repeated. If $\mathrm{M}^{-1 / 2} \leq \mathrm{C}$ is not satisfied, then the current returning through the side layers parallel to the applied field is not negligible, and is included in the analysis with respect to $c$ and $M / 2 /$ In this 
case the variables in the core must be adjusted considering the higher volume flux through the side layers.

This method is valid only if a significant portion of the magnetic field is perpendicular to the duct, particularly $\mathrm{B}_{\mathrm{y}}=0$ is excluded. As mentioned above, the Hartmann number and interaction parameter need to be high. Moreover the geometry must be smooth, abrupt expansions or contractions require higher $M$ and $N$ for the solution to be valid. For any more complex geometry, like bends and smooth variation of the duct's cross section, the analytical integration along field lines becomes more and more expanded. Due to the fact that viscosity is neglected, the values of the velocities in the thin shear layers are not exact, and corrosion analysis is not possible with CFS.

As a result of the algorithm both, the storage and the computational time needed, is not a real restriction compared to the FS. The typical cpu-time for the magnetic field [3.1], a rectangular duct and $90 \times 30$ grid points, is less than 10 minutes. 


\section{Numerical results}

One incisive restriction of the CFS is that high Hartmann numbers and interaction parameters are presumed. It is important to know how high these numbers must be, in order to estimate the range of validity of this approximate method. As an example a straight rectangular duct ( see figure 1 on page 5 ) with a constant wall conductance ratio of $\mathrm{c}=0.07$ and an applied magnetic field specified in [3.1] is investigated. The results of FS and CFS are compared at Hartmann numbers of $M=50,100,200,250,300$ and several interaction parameters. To find out the influence of $M$, being characteristic of the magnitude of viscous effects, the interaction parameter is kept constant at $N=2000$ first. This value satisfies condition [3.2] for all the Hartmann numbers checked up here. In the second part of the computations, flow variables with a constant Hartmann number and various interaction parameters are investigated.

Figures 2 - 4 show curves of the dimensionless pressure (dashes), the velocity in $x$-direction ( chaindots) and the electrical potential (dots) against the axial $x$-direction of the duct for FS and CFS. The applied magnetic field ( solid curve) is plotted for reference. The values for $p, \Phi$ and the velocity component in $\mathrm{x}$-direction are given at the axis of the duct, at $y=0$ and $z=0$, and close to the middle of the side wall, at $y=0$ and $z=0.92$. These are positions of the cross section where the variables differ very much. There is a slight difference between the chosen coordinates of the FS and the CFS $(\sim 0.02)$, because the discretisation in the two numerical codes is not exactly the same. This has little effect on the comparison.

The MHD flow in all the cases presented here has three distinct regions. The first region, where $B_{y}$ is constant at its maximum value of $B_{y}=1$, the second where it slowly decreases, and a third with $B_{y}$ down to its minimum value, being constant again. A minimum magnetic field of $B_{y}=0.05$ is applied, because $B_{y}=0$ is not allowed for the CFS. Area one and three have some similarities. The velocity ( chaindots ) and the potential (dots ) are constant in the $x$-direction there. Figures 2 - 4 point out a constant pressure gradient in the axial direction for these two fully developed regions. The pressures in the middle and the side are exactly the same. The whole MHD flow is two-dimensional.

Calculation with the FS-code from $x=-10$ to $x=10$ is sufficient then to save computer storage. 

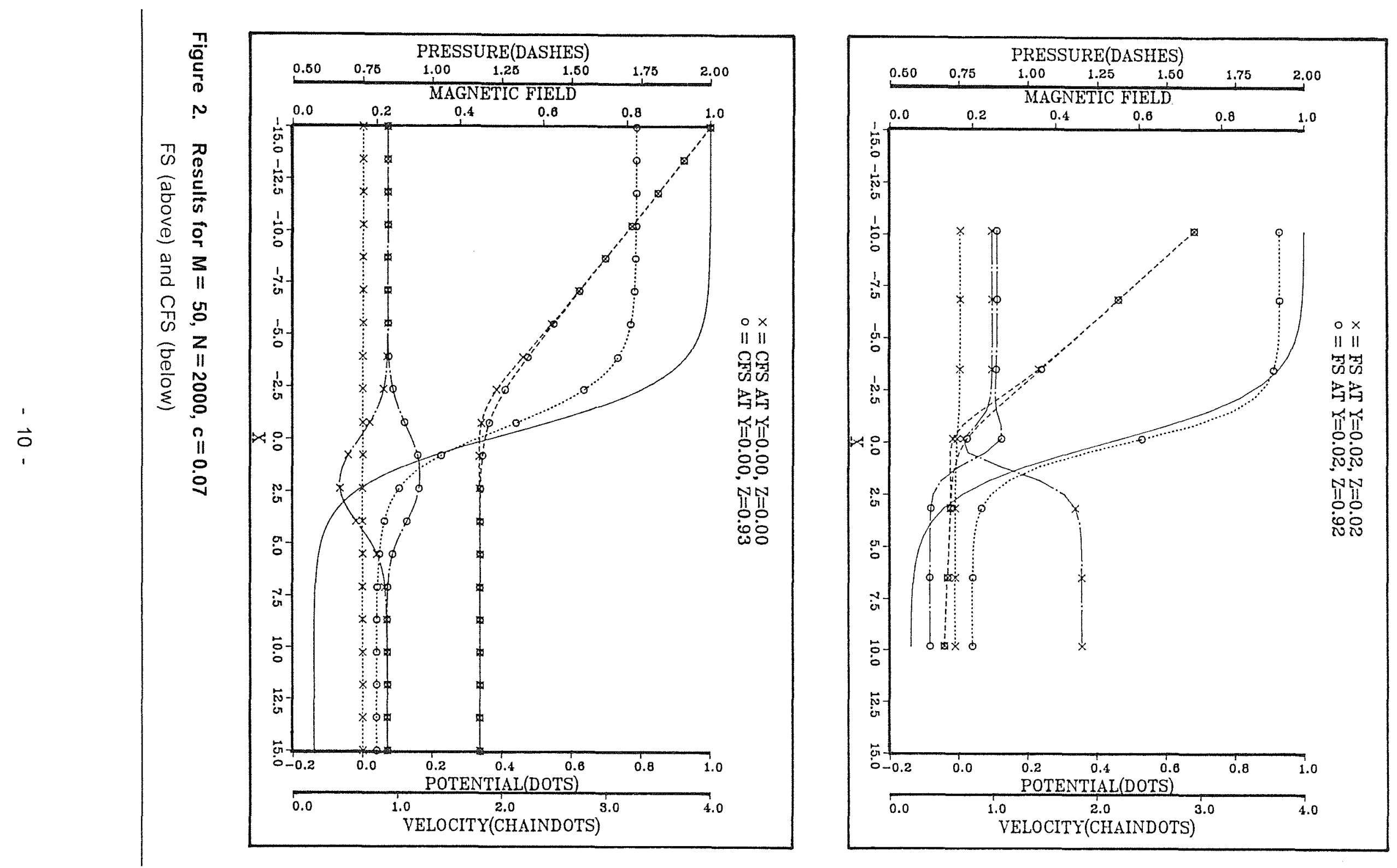

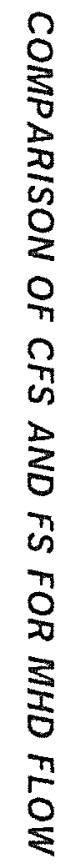



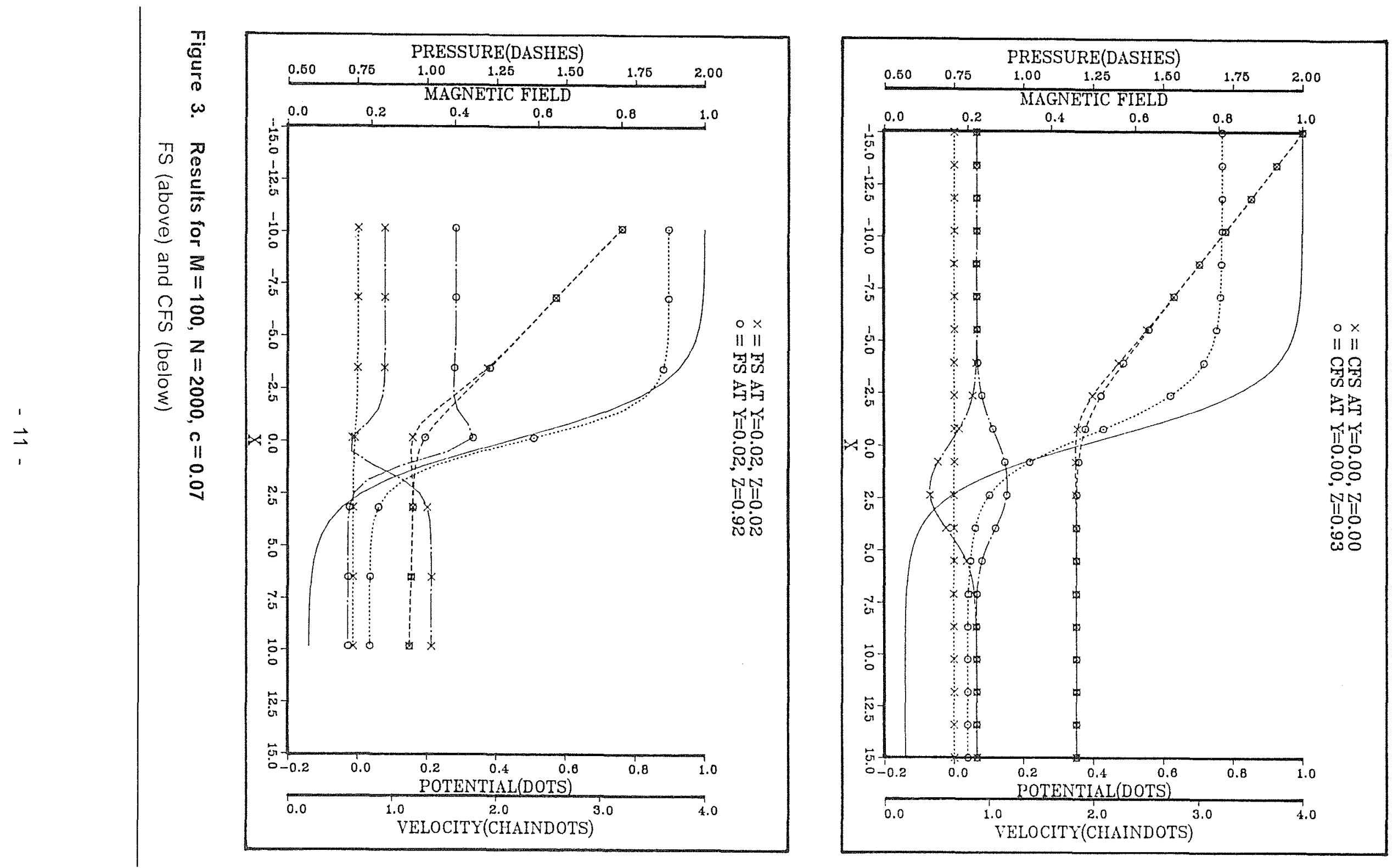

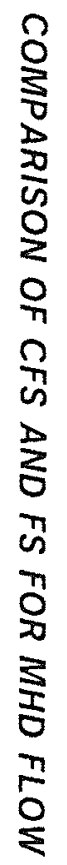



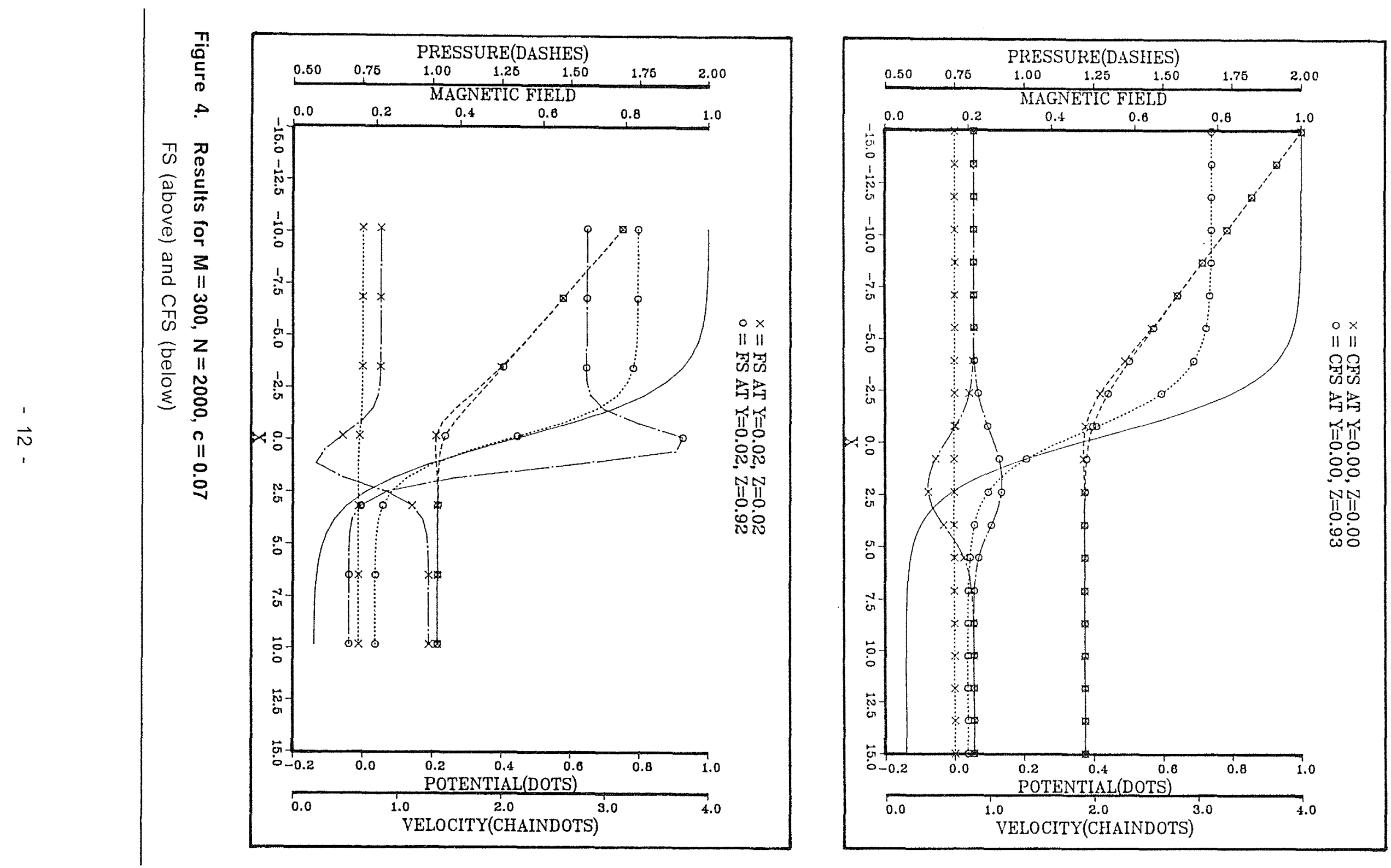

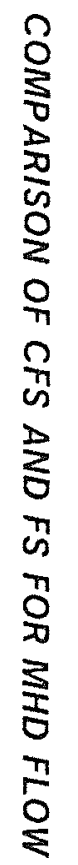


One difference between the results of the two methods can be detected at the duct outlet at $x>5$. For the CFS the pressure gradient vanishes when the magnetic field is nearly zero, whereas the FS still predicts a small pressure gradient, because this method accounts for the viscous effects.

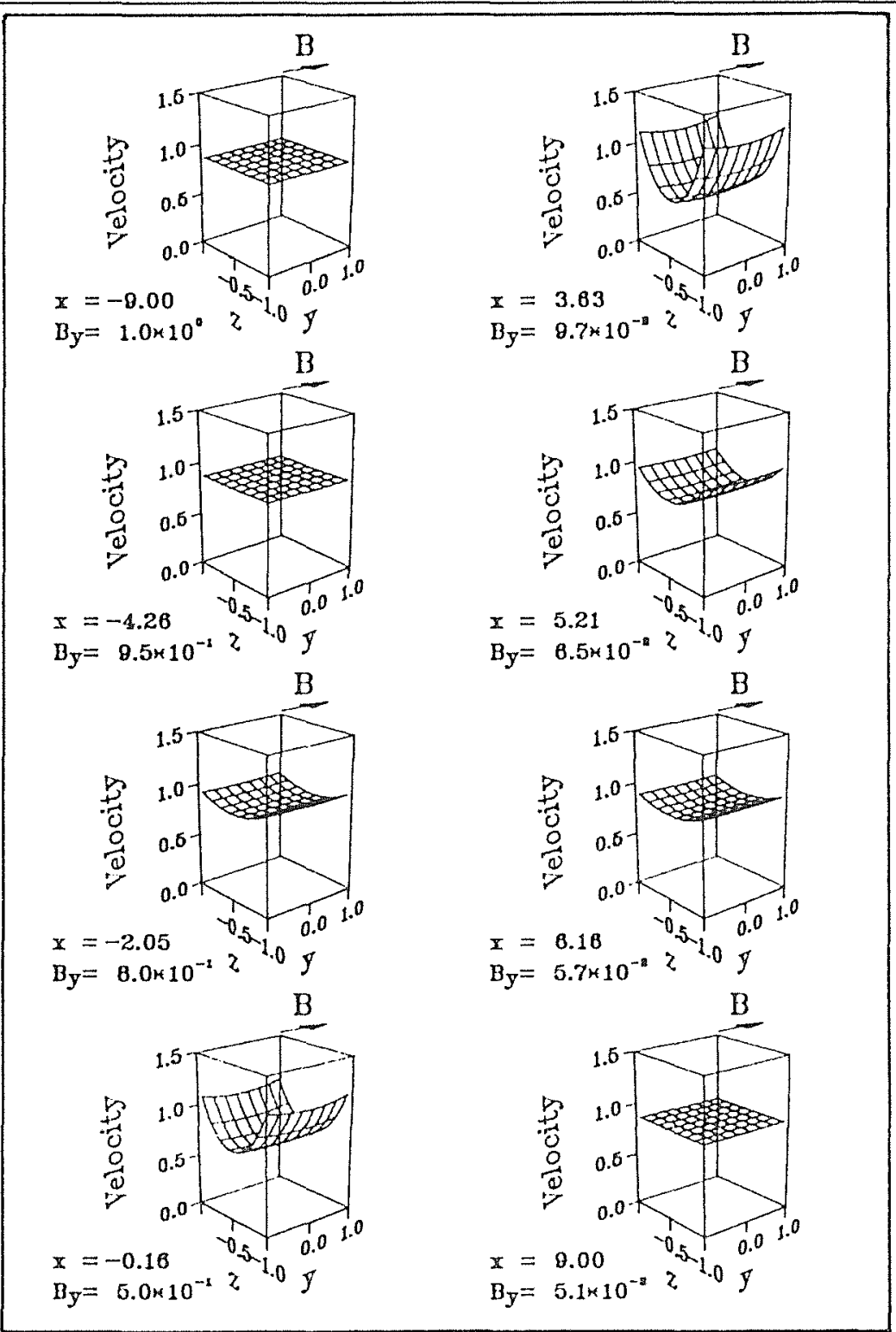

Figure 5. Development of velocity distribution for CFS (3-D plot) $M=200, N=2000, c=0.07$

The inertial effects do not appear in the fully developed flow. There is no timedependance under steady state conditions and no $y$ - or z-component of the velocity. Suprisingly, it seems as if the viscous pressure gradient is higher for a lower Hartmann number ( compare figure 2 and figure 4 for example). But 
these are nondimensionalized numbers, and for equal volume fluxes the pressure has to be multiplied by $\mathrm{M}^{2}$ for a comparison. Then it can be seen that the resulting pressure gradient is not higher for lower $M$. The velocities obtained from the CFS-code turn out to be the same in the middle of the duct and at $z=0.93$, as long as $B$ does not change. Figure 5 displays this result. Velocity distributions are presented for several cross sections along the duct axis. The magnitude of the magnetic field and the corresponding $x$-coordinate is given below each subplot.

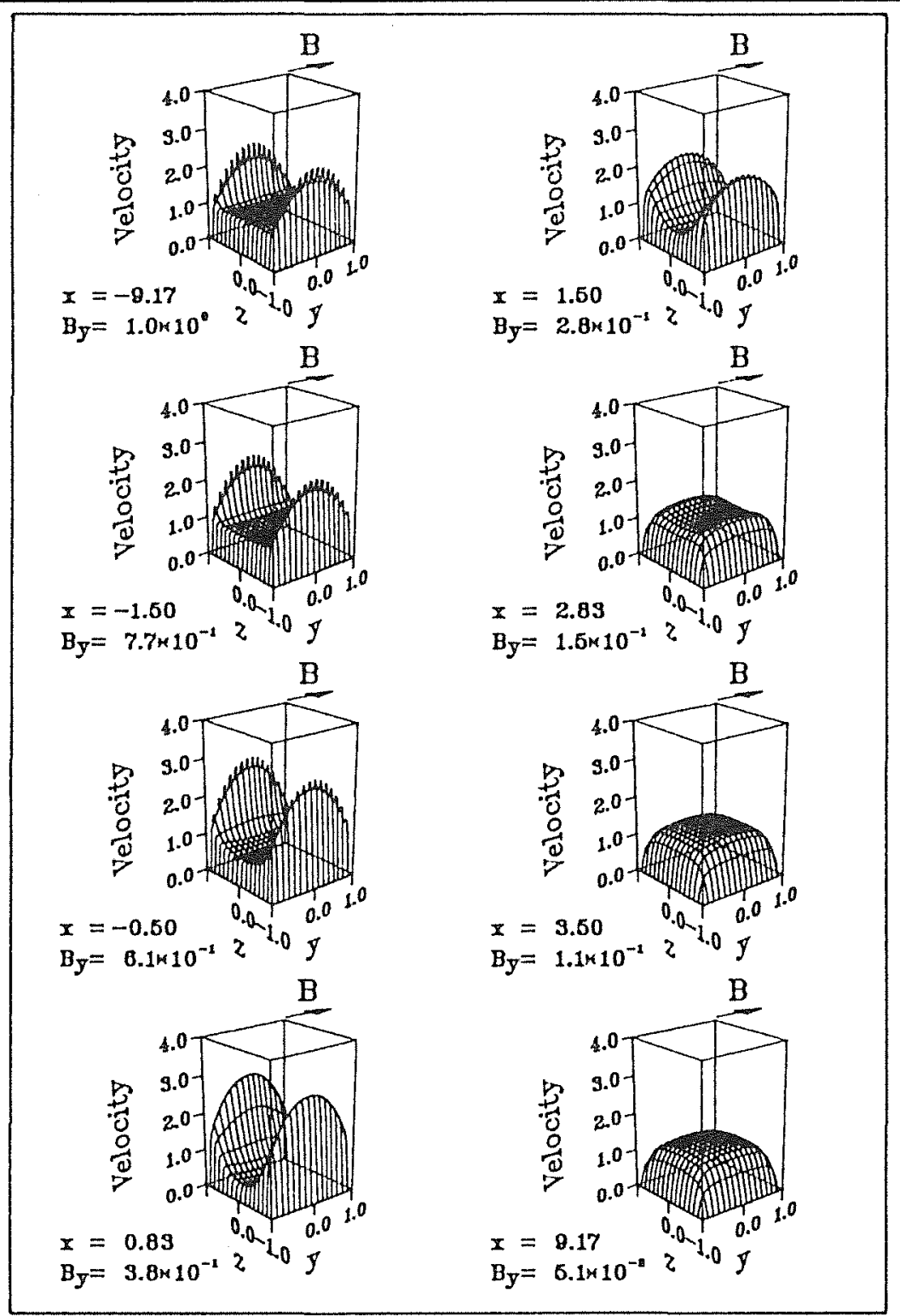

Figure 6. Development of velocity distribution for FS (3-D plot) $M=200, N=2000, c=0.07$ 
Lorentz forces are uniform in the core as long as $B_{y}=1$ is constant. The CFS-code calculates the core variables only, and therefore yields a flat velocity distribution in this regions. Figure 6 shows the results for the FS. With
$B_{y}=1$ the typical M-shaped velocity profile of fully developed viscous MHD flow can be seen. The no-slip condition is satisfied at the walls, in contrast to the CFS.

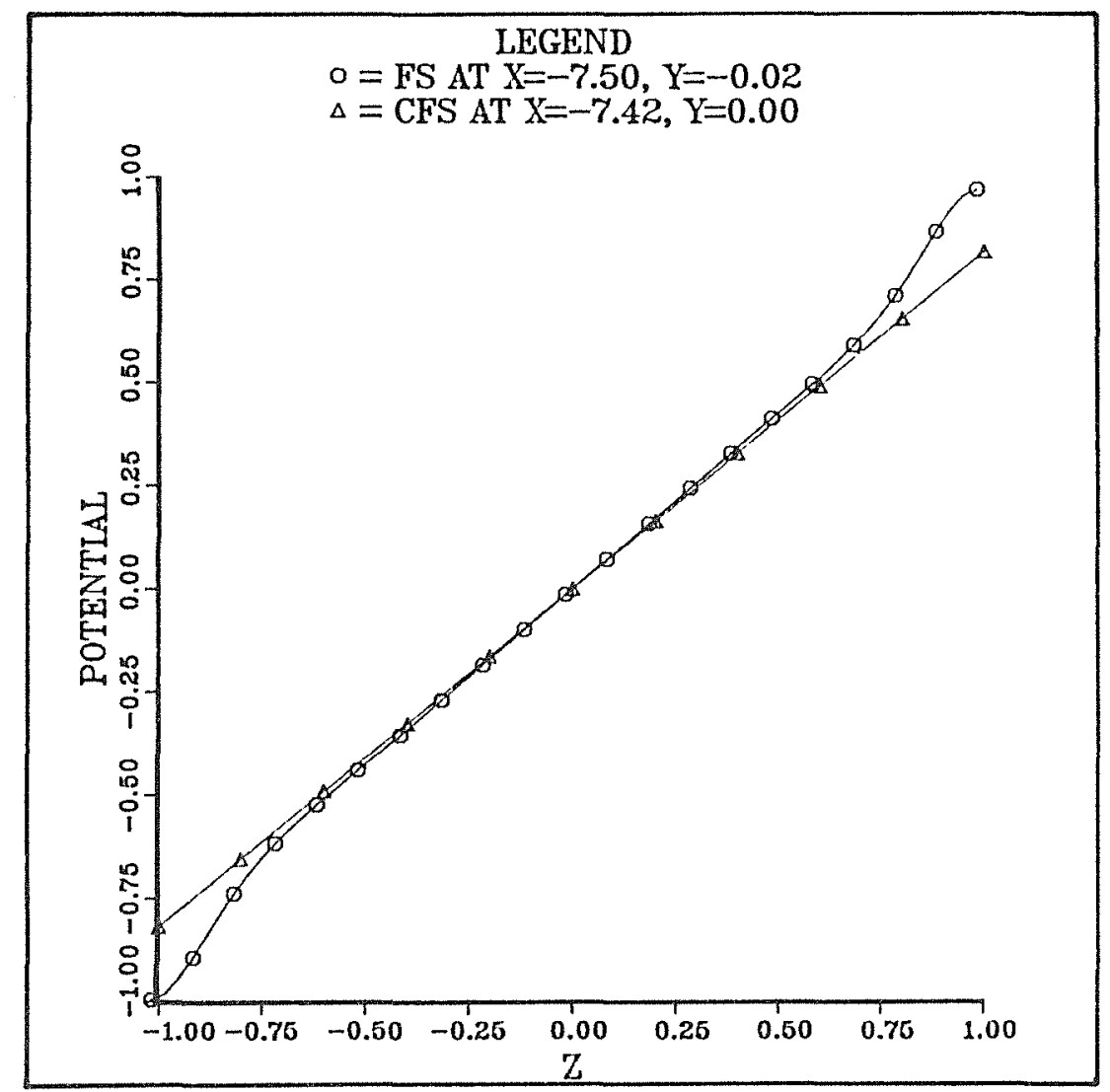

Figure 7. Potential in the $y-z-p l a n e$ at $y=0$ and $x=-7.5$

$M=100, N=2000, c=0.07$

An ordinary hydrodynamic profile results downstream for $x>3$, where the magnetic field is very low. Figures 2- 4 again demonstrate the differences between the two solutions there. In the FS the velocity changes from a higher value in the side layer at $x<-3$ to a lower one at $x>3$, in accordance with the $M$-shape profile and the nearly hydrodynamic profile, repectively. Once more the CFS shows uniform velocities for $x<-3$ and $x>3$. For high Hartmann numbers and interaction parameters these differences between the two solutions only accur at the boundary layers. As mentioned before, these layers are thin and decrease with M. A good estimation of the vol- 
ume flux in the side layers is possible with respect to the wall conductance ratio and the Hartmann number $/ 2$ /. For these reasons the CFS cannot be taken for corrosion analysis, but yields good predictions of the heat transfer.

The development of the potential in axial direction show a similar behaviour for FS and CFS. As a symmetry condition it can be adjusted to zero in the axis of the duct, at $z=0$. Potential differences between the middle and the side are reduced as the magnetic field decays. The potential difference at $x<-5$ where the flow is fully devel- oped is about 17 percent higher for the FS at $M=50$, falling down to 9 percent at $M=300$. This is due to the fact that the CFS does not include the calculation of the potential jumps across the side layer. The potential distributions given in figures 2 - 4 show the different results for these side layers, whereas the potential in the core of the duct show a very good agreement of both methods ( see figure 7 ). For higher Hartmann numbers a lower influence of the viscosity and a lower difference in the potentials is expected, and figures $2-4$ show this trend in the side layers.

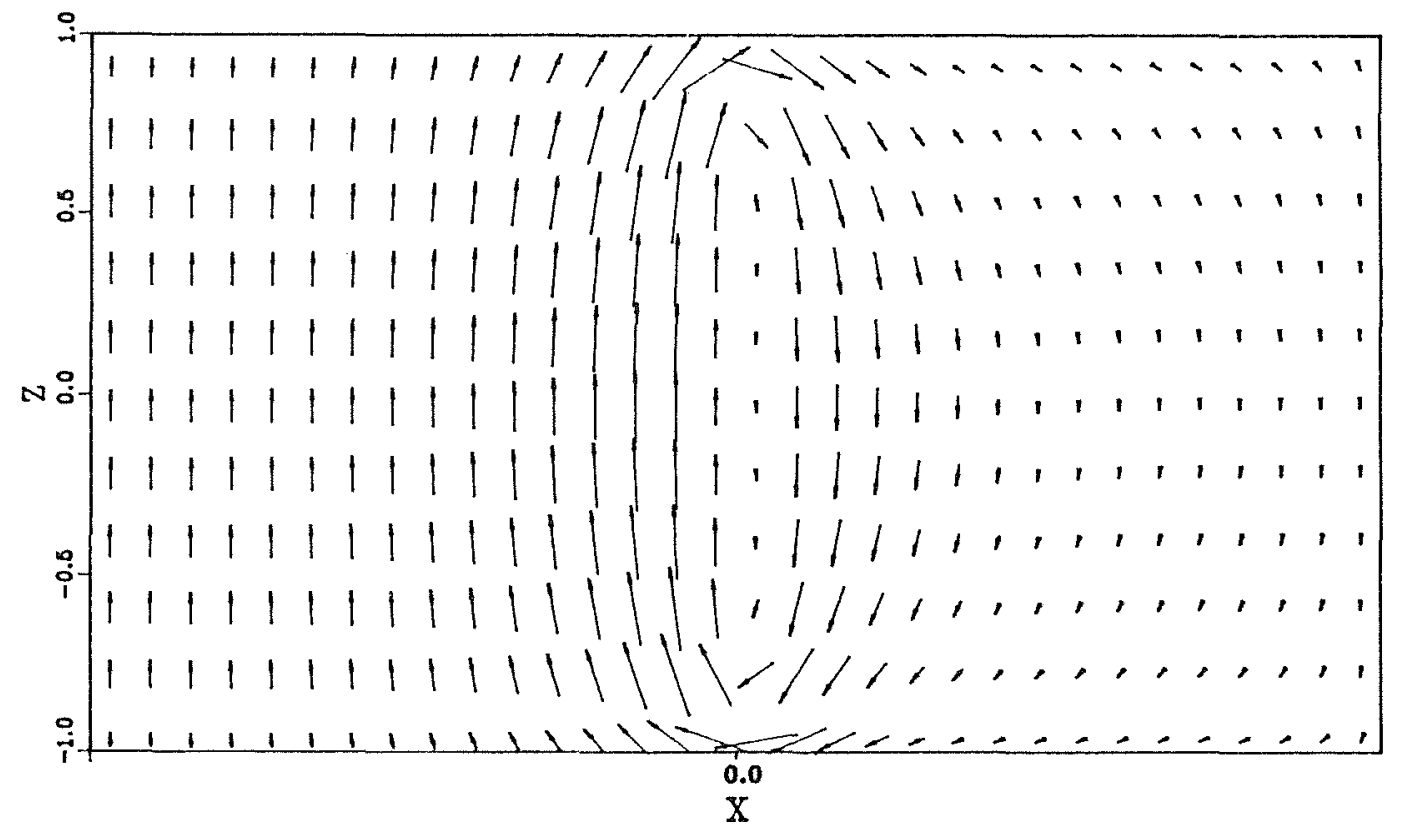

Figure 8. Current in the $x-z-p l a n e$ at $y=0$ $M=50$, schematic

Three-dimensional effects can be seen in region two, where the magnetic field slowly decreases. The lower down- stream magnetic field induces lower potential differences between the side and the center of the duct. Due to the 
resulting axial potential differences axial currents are induced. They are responsible for Lorentz forces, which have to be balanced by a pressure gradient. This results in a pressure difference seen in figures $2-4$, where the pressure is higher close to the side wall in this $3-d$ region. Here FS and CFS have very similar results. More- over these axial current must have a short circuit due to conservation of electric charge. This short circuit is in the $x$-z-plane ( see figure 8 ). The additional z-component of the current is higher in the core than close to the side walls. Subsequently there is a velocity jet in the side layers.

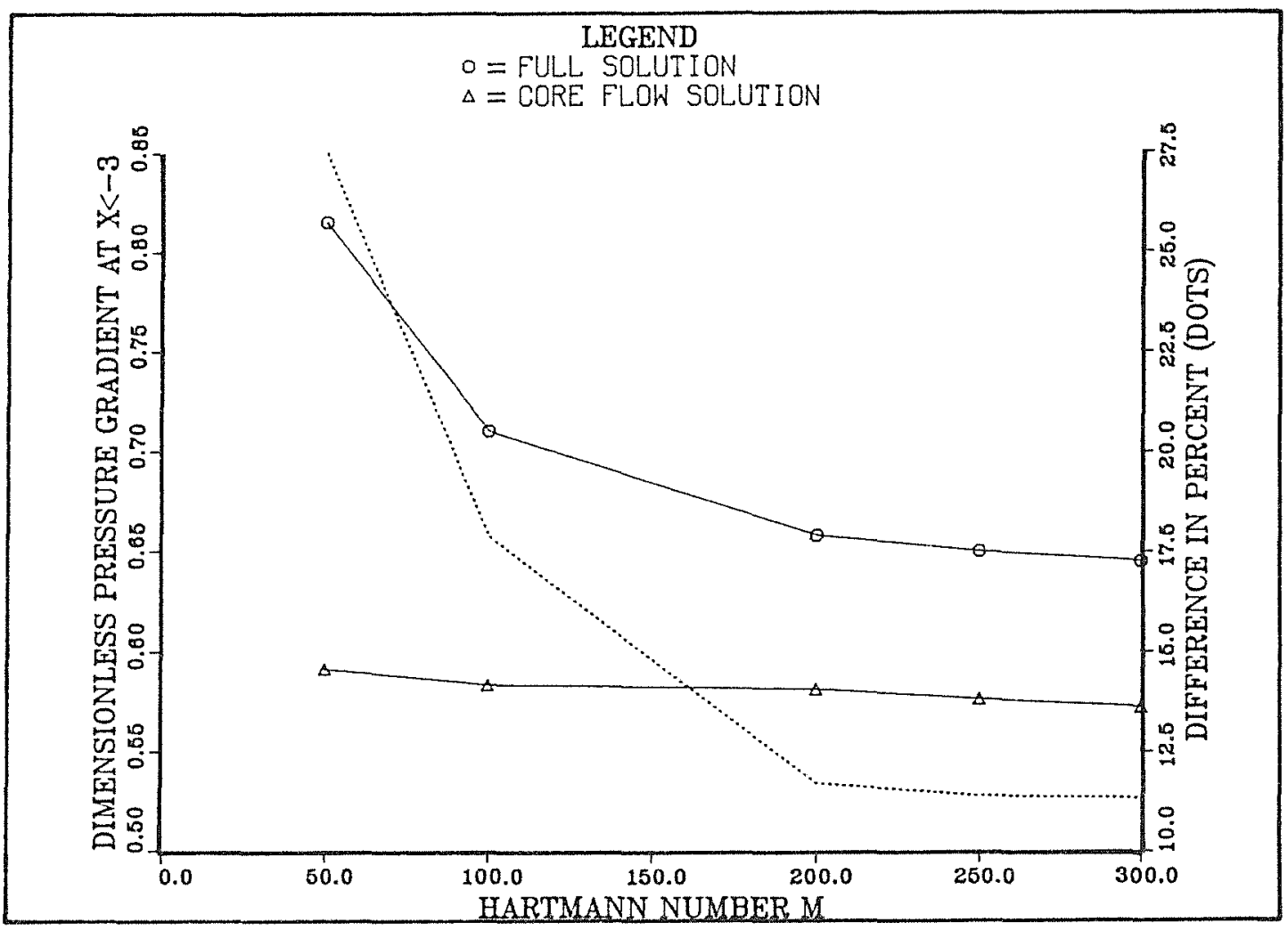

Figure 9. Comparison of the pressure gradient

Pressure gradient of FS and CFS in the entrance of the duct, where the magnetic field is constant $(B=1)$

Again FS and CFS show the same slow down in the middle of the duct. trend, but for the reasons mentioned The whole flow is driven towards the above the magnitude of these jets is side layers in the decreasing field. much higher in the FS. To satisfy conFigure 6 shows the effects in more servation of mass, the velocity must detail for the FS. The flow has the 
expected fully developed $M$-shape at $\mathrm{x}$ $=-9.17$, is driven to the side layers in the 3 - $d$ region and forms an ordinary hydrodynamic profile at $x>3$. Figure
6 indicates the additional volume flux through the sides in the varying field for the FS.

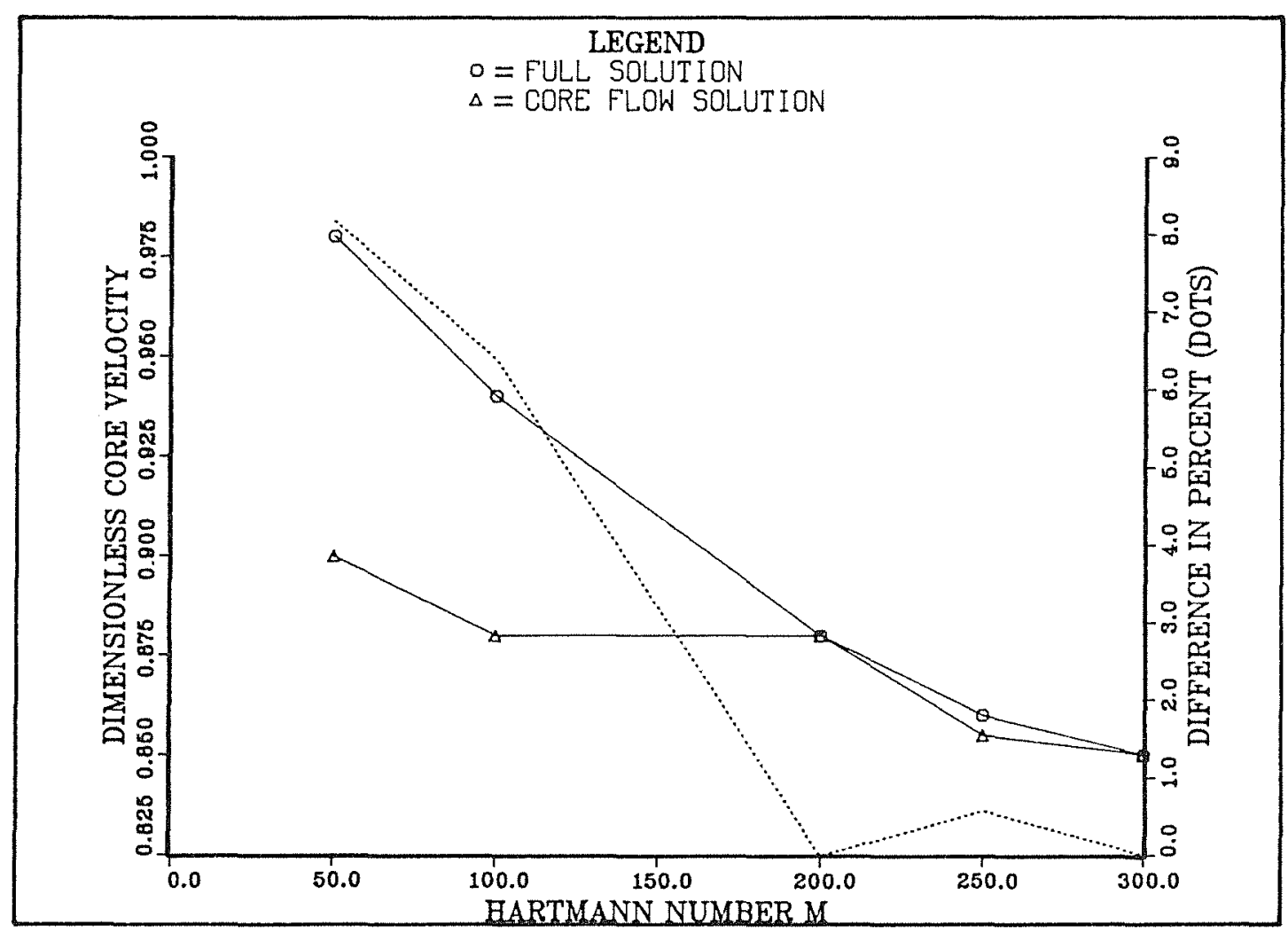

Figure 10. Comparison of the core velocity

Core velocity of FS and CFS in the entrance of the duct, where the magnetic field is constant $(B=1)$

Comparison of the axial pressure gradients in the fully developed region with $B_{y}=1$ provides another possibility to check the quality of the calculations. Figure 9 demonstrates these pressure gradients for FS and CFS with Hartmann numbers between 50 and 300 and an interaction parameter of 2000 . The third curve in this plot shows the difference between the FS and CFS in percent. Viscous effects considered in the $F S$ require a higher pressure difference between the inlet and outlet of the duct ensuring the same volume flux as the CFS. It seems as if the pressure gradient decreases at higher Hartmann numbers. The reason for this is the dimensionless form the the pressure gradient. Of course the pressure losses are enhanced at 
higher $M$. On the other hand, the difference between the FS and the CFS is reduced down at higher $M$, as expected, because viscosity becomes less and less important. For $M>100$ the agreement is within acceptable error limits.

The core velocity is compared in the same region, too. Figure 10 illustrates this core velocity for the FS and CFS with Hartmann numbers between 50 and 300 and $N=2000$. Their difference in percent is also shown. The values obtained from the CFS match very well to Walker's asymptodic solution valid for $M^{-2} \ll c \ll \sqrt{M} / 8 /$.

This relation is not satisfied for Hartmann numbers less than about 100 , if the wall conductance ratio is $\mathrm{c}=0.07$. Then the the currents closing through the side layers are not negligible. The same result can be maintained from figure 10 . For $M \geq 200$ the agreement between FS and CFS is within one percent. Results for $M \leq 100$ show the expected difference, but even for $M=$ 50 the percentage is still less than nine.

In the three-dimensional region and at the transition from three- to two-dimensional flow inertial effects might affect the redistribution of the flow. In order to investigate this effect, several interaction parameters are combined with a constant Hartmann number of $M=100$. Figure 11 when compared to figure 3 shows that there is no significant difference between the flow variables at $N=200, N=1000$ and $N==2000$. The same is true for any higher interaction parameter.

Of course inertial effects will be important for sufficiently low interaction parameters. This could be investigated using this FS-code only on computers with a larger storage than available today, due to the lower limit on $N$ required by the restriction on mesh Reynolds number.

A more general influence of inertial terms can be detected in figures $2-4$. In the CFS the variation between velocities in the center and near the sidewalls starts slightly further upstream than in the FS. This is because the modell for the current returning through the side layers used in the CFS is not valid if the magnetic field changes. But for slowly varying fields it still yields acceptable results / $2 \%$ 

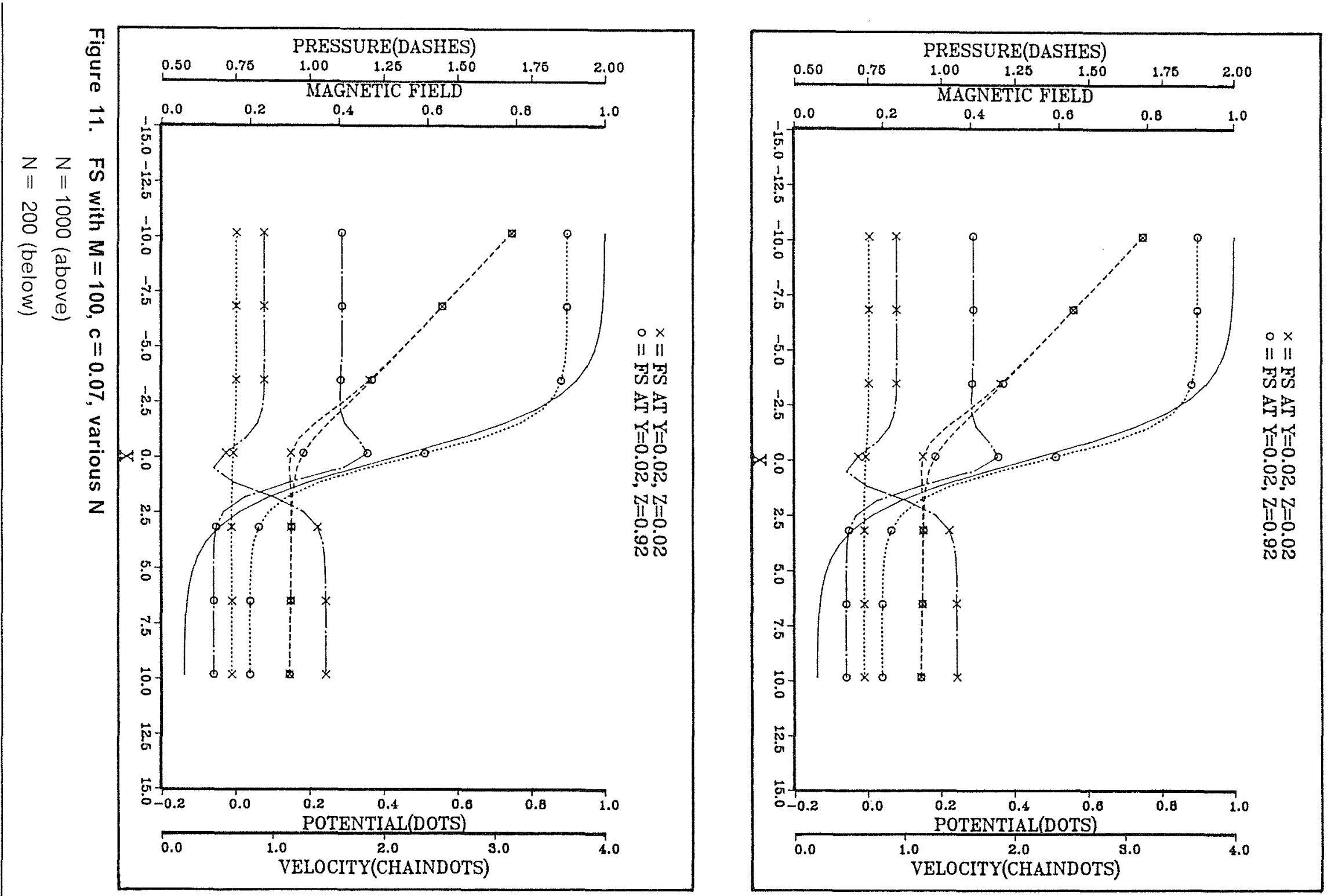

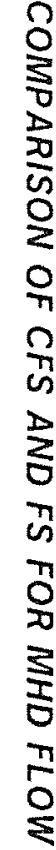




\section{Conclusions}

The core flow solution and the full solution are compared for a rectangular duct with a wall conductance ratio typical for fusion reactor blankets under the influence of a variable transverse magnetic field. The difference of the calculated pressure gradient in the fully developed region is about 27 percent for Hartmann number of $M=50$, decreasing to about 11.5 percent for $M=300$. The potential distributions calculated with the two methods agree very well exept for the thin side layers. The core flow velocities show an even better agreement. Their difference is negligible for $M>100$, i.e. less than 1 percent. It is reasonable to transfer these results to any straight duct with similar wall conductance ratio under constant or slowly varying fields.

The mesh Reynolds number limits the lower values of the interaction parameters $N$ used in the calculations for the full solution. All the interaction parameters parameters studied here, $\mathrm{N}>$ 100 for $M=50$ and $N>2000$ for $M=300$, do not effect the results significantly. Further comparisons should be done for lower interaction parameters and more complex geometries such as bends and sudden expansions, where inertial forces and/or viscosity might have a greater effect. 


\section{Appendix A. Nomenclature}

All nondimensionalized variables have a o as a subscript. The typical numbers set in parentheses are from / 10, $11 /$.
$x, y, z$
cartesian coordinates
a
half width of the $(\sim 0.3 \mathrm{~m})$
$t_{w}$
wall thickness $(\sim 0.001 \mathrm{~m})$
B
magnetic field (3-5 tesla)
M
Hartmann number $\left(10^{3}-10^{4}\right)$
$N$
interaction parameter $\left(10^{2}-10^{4}\right)$
$\mathrm{Re}_{\mathrm{m}}$
magnetic Reynolds number $(\ll 1)$
c
wall conductance ratio $\left(10^{-2}-10^{-3}\right)$
$\eta$
viscosity $\left(10^{-4}-10^{-3} \mathrm{Ns} / \mathrm{m}^{2}\right)$
$\sigma$
electric conductivity $\left(\sigma_{\text {fluid }} \sim 10^{6} 1 / \Omega \mathrm{m}\right)$
$\rho$
density $\left(\rho_{\text {fluid }} \sim 10^{3} \mathrm{~kg} / \mathrm{m}^{3}\right)$
velocity (average velocity $\sim 0.5 \mathrm{~m} / \mathrm{s}$ )
j
current density
$\Phi$
electric potential
$\mathrm{p}$
pressure

V 


\section{Literature}

1. A. Sterl

Numerische Simulation magnetohydrodynamischer FlüssigMetall-Strömungen in rechteckigen Rohren bei großen Hartmannzahlen

KfK-Bericht Nr. 4504, 1989

2. K. McCarthy

Analysis of Liquid Metal MHD Flow Using a Core Flow Approximation, with Applications to Calculating the Pressure Drop in Basic Geometric Elements of Fusion Reactor Blankets

Dissertation, University of California, Los Angeles, February 1989

3. L. Barleon, L. Lenhart, H.J. Mack, A. Sterl, K. Tomauske Investigations on Liquid Metal MHD in Straight Ducts at High Hartmann Numbers and Interaction Parameters

Proceedings of the NURETH-4 Meeting, Karlsruhe, October 10-13, 1989, pp. 857-862
4. B.F. Picologlou, C.B. Reed, T.Q. Hua, L. Barleon, H. Kreuzinger, J. Walker

Experimental Investigations of MHD Flow Tailoring for First Wall Coolant Channels of Self-Cooled Blankets

Eighth Topical Meeting on the Technology of Fusion Energy, Salt Lake City, Utah, October 9-13, 1988

5. D.A. Anderson, J.C. Tannhill, R.H. Plether

Computational Fluid Mechanics and Heat Transfer

Hemisphere Publishing Corporation, New York, 1984, pp. 343-346

6. T.Q. Hua, J.S. Walker, B.F. Picologlou, C.B. Reed

Three-Dimensional Magnetohydrodynamic Flows in Rectanguiar Ducts of Liquid-Metal-Cooled Blankets

Fusion Technology, Vol. 14, 1988 , pp. $1389-1398$ 
7. J.A. Shercliff

A Textbook of Magnetohydrodynamics

Pergamon Press, Oxford, 1965
Applications of Laboratory and Theoretical MHD Duct Flow Studies in Fusion Reactor Technology UKAEA-Report, CLM-R 169, 1977

10. R.J. Holroyd, J.T.D. Mitchell Liquid Lithium as a Coolant for Tokamak Fusion Reactors Nuclear Engineering and Design/Fusion, Vol. 1, 1984, pp. 17-38.

11. D.L. Smith

Blanket Comparison and Selection Study - Final Report

ANL-Report, ANL/FPP 84-1, Argonne National Laboratory, 1984 
ACKNOWLEDGEMENT

This work has been performed in the framework of the Nuclear Fusion Project of the Kernforschungszentrum Karlsruhe and is supported by the European Communities within the European Fusion Technolgy Program. 